\title{
$\Delta=$
}

\section{RASTROS DOS PRIMEIROS CURSOS DE FORMAÇÃO DE PROFESSORES DE MATEMÁTICA NA CIDADE DE UBERLÂNDIA - MINAS GERAIS}

\section{TRACES OF THE FIRST MATHEMATICS TEACHER TRAINING COURSES IN THE CITY OF UBERLÂNDIA - MINAS GERAIS}

Douglas Marin $^{1}$

\begin{abstract}
RESUMO
Este artigo apresenta um estudo cujo cenário de investigação são os primeiros cursos de formação de professor de Matemática na cidade de Uberlândia. Esta pesquisa está integrada a uma maior que trata da formação de professores de Matemática no Triângulo Mineiro. Com o intuito de compreender como foi o processo de criação dos cursos de formação de professores de Matemática na cidade de Uberlândia nas décadas de 1960 e 1970 , utilizamos como fonte de dados, além das edições da Revista Documenta que foram publicadas nos anos de 1962 até 1979 e outros documentos disponíveis nos auxiliaram nesse processo. Os resultados dos dados mostraram a existência de dois cursos de formação de professores de Matemática em duas instituições de ensino superior nessa localidade no período especificado. As expectativas de contribuição deste trabalho são para a formação inicial de professores de Matemática e para História da Educação Matemática.
\end{abstract}

PALAVRAS-CHAVE: História da Educação Matemática. Formação de Professores de Matemática. Educação Matemática.

\begin{abstract}
This article presents a study whose research scenario are the first mathematics teacher training courses in the city of Uberlândia. This research is integrated to a greater dealing with the training of mathematics teachers in the Triangulo Mineiro. In order to understand how was the process of creating mathematics teacher training courses in the city of Uberlândia in the 1960s and 1970s, used as a source of data, in addition to issues of the magazine Documents that were published in the years 1962 to 1979 and other documents available in assisted this process. The results of the data showed the existence of two mathematics teachers training courses at two institutions of higher education in this location in the specified period. The contribution of this work expectations are for initial training of mathematics teachers and History of Mathematics Education.
\end{abstract}

KEYWORDS: History of Mathematics Education. Mathematics Teacher Education. Mathematics Education.

\section{INTRODUÇÃO}

A história da formação de professores de Matemática tem sido foco de diferentes estudos realizados no Brasil, e ganha abrangência nas pesquisas desenvolvidas pelo Grupo de História Oral e Educação Matemática - Ghoem². Em um dos principais projetos desse grupo

\footnotetext{
1 Universidade Estadual Paulista "Júlio de Mesquita Filho" - UNESP (Rio Claro). E-mail: douglasmarin2007@gmail.com

${ }^{2 " O} \mathrm{O}$ GHOEM é um grupo de pesquisa interinstitucional que congrega profissionais de diversos estados brasileiros. Seus membros são pesquisadores em Educação Matemática e seus orientandos de mestrado e doutorado. Mantendo-se no grupo, esses mestrandos e doutorandos passam a orientar trabalhos e agregam ao GHOEM seus orientandos." (GARNICA, FERNANDES; SILVA, 2011, p. 230) Para mais detalhes visite http://www.ghoem.org. < Acessado em maio de 2015>.
} 
está o objetivo de elaborar um mapeamento sobre a formação e atuação de professores de Matemática no Brasil.

A preocupação destas pesquisas com a formação de professores é latente, mas, também, voltam seus olhares para as práticas no fazer pedagógico nas diversas modalidades de ensino, na institucionalização de cursos que formavam professores (de Matemática), na formação da identidade de grupos de pesquisa, no traçado do perfil de professores e alunos de distintas regiões, nas motivações políticas, administrativas e sociais, entre outros vieses.

Esse enfoque pode ser constatado em pesquisas como em BARALDI (2003), PINTO (2006), CURY (2007; 2011), MARTINS-SALANDIM (2007; 2012), FILOS (2008), FERNANDES (2011), MORAIS (2012), FERREIRA, BRITO e MIORIM (2012), TROILLIER (2013), MACENA (2013), BOTH (2014), FAORO (2014), GARNICA (2014) SILVA (2015), ALMEIDA (2015) e MARIANO DA SILVA (2015).

O mosaico de pesquisas apresentado mostra-se como um solo bastante fértil para apoiar esse estudo. Considerando que assumimos uma determinada postura historiográfica segundo a qual o estudo do passado do processo educativo deve ser disparado pelo presente visando a colaborar com o presente, trazendo à tona pressupostos, práticas, atitudes e preconceitos, entendemos ser altamente importante para a Educação, para Educação Matemática, para o Triângulo Mineiro, para a História da Educação e da História da Educação Matemática.

Este estudo pretende inserir-se no que atualmente tem se chamado de História da Educação Matemática, na medida em que se propõe a compreender, analisar, elaborar, discutir a reconstituição de histórias dentro da própria História (GARNICA, 2013) e dispõe-se a delinear um mapeamento da formação de professores (de Matemática) do no Triângulo Mineiro.

Com o intuito de contribuir com essa área, neste texto, apresentamos uma síntese de reflexões iniciais, com foco na cidade de Uberlândia, cujo cenário são os primeiros cursos de formação de professores de Matemática, nas décadas de 1960 e 1970.

Nesse período, constatamos a criação de dois cursos. O primeiro deles, atestado pelo Decreto $\mathrm{n}^{\circ} 60 / 71^{3}$ de 30 de maio de 1967 onde foi concedido a Faculdade de Filosofia, Ciências e Letras da Fundação Universidade de Uberlândia a autorização para funcionar o curso de Matemática e, o segundo, verificado na Revista Documenta, n 147, de 1973 a

${ }^{3}$ Documenta n ${ }^{\circ} 72$, p. 32, 1972. 
autorização de uma Faculdade de Educação mantida pela Associação Brasil Central de Educação e Cultura. Sendo que poucos anos depois constatamos pelo Decreto $\mathrm{n}^{\mathrm{o}} 76.064 / 75^{4}$ foi concedida a autorização para funcionar o curso de Ciências, sendo o curso de Matemática uma de suas habilitações.

Para dar suporte as reflexões e discussões dos resultados, ora apresentadas, trazemos, no que segue no texto, um resumo dos aportes teóricos pautados na História da Educação Matemática que conduz essa pesquisa.

\section{História}

Com esta pesquisa, busco compreender e elaborar versões históricas, segundo as narrativas constituídas por meio de entrevistas, bem como de perspectivas documentais, de como se deu o processo de formação de professores que ensinaram Matemática, entre as décadas de 1960 a 1990, a partir dos primeiros cursos de formação superior, no Triângulo Mineiro, Minas Gerais. Nesse sentido faz-se necessário discutir algumas acepções de "História" haja vista a multiplicidade de significados atribuídos a esta palavra.

Inicialmente, ao procurar a verbete "História" no dicionário Priberam", encontro que se trata de uma "narração escrita dos factos notáveis ocorridos numa sociedade em particular ou em várias; Ciências ou disciplina que estuda factos passados, livro de história, estudo das origens e progressos de uma arte ou ciência".

No entanto, ao questionar alguém sobre o que é História, poderia receber algumas respostas previsíveis como, por exemplo: "uma forma de estudar o passado", ou "a ciência que investiga o passado da humanidade e o seu processo de evolução".

Após uma busca mais sofisticada, num dicionário de Filosofia, lemos que

esse termo, que em geral significa pesquisa, informação ou narração e que já em grego era usado para indicar a resenha ou a narração dos fatos humanos, apresenta hoje uma ambiguidade fundamental: significa, por um lado, o conhecimento de tais fatos ou a ciência que a disciplina e dirige esse conhecimento e, por outro, os próprios fatos ou um conjunto ou a totalidade deles (ABBAGNANO, 2007, p. 583).

\footnotetext{
${ }^{4}$ Documenta $^{\circ}$ 176, p.381, 1975.

${ }^{5}$ PRIBERAM, disponível em <www.priberam.pt/dlpo/>
} 
Na tentativa de trazer outras acepções sobre História trago Albuquerque (2011, p. 173) segundo o qual

a História seria movimento, seria ação criativa, invenção constante de novos lances, mesmo que seus sujeitos estejam limitados por regras, por normas, tenham que obedecer a regulamentos. A História é possível porque os homens, mesmo limitados por um dado contexto, por um conjunto de regras e prescrições, mesmo atuando em um espaço e um tempo delimitado, são capazes de driblar a potência do mesmo e a imposição de repetição e criar o diferente, a novidade, de produzirem a surpresa e o inesperado. A História, como jogo, faz-se de risco e habilidade, de variação e mudança, de limite e invenção, de regras imanentes e de restrições voluntárias.

Ao ver a História como uma forma de expressar algo do passado a partir do presente, compreendido por meio de indícios que podem ser encontrados em fontes várias naturezas (orais, escritas, arquitetônicas etc) nos possibilita criar versões plausíveis de um passado.

Garnica; Souza (2012, p.21), baseados em Marc Bloch, têm defendido a concepção de que a História, de forma geral, é "uma ciência dos homens no tempo e espaço. Mas como não se vive só, e sim, em comunidade, poderíamos enunciar a concepção de História [...] como: a História é o estudo dos homens vivendo em comunidade no tempo".

A história é, então, uma construção sem relação ao mundo em si. Mais especificamente:

A História é um discurso cambiante e problemático, tendo como pretexto um aspecto do mundo, o passado, que é produzido por um grupo de trabalhadores cuja cabeça está no presente (e que, em nossa cultura, são na imensa maioria trabalhadores assalariados), que tocam seu ofício de maneiras reconhecíveis uns para os outros (maneiras que estão posicionadas em termos epistemológicos, metodológicos, ideológicos e práticos) e cujos produtos, uma vez colocados em circulação, vêem-se sujeitos a uma série de usos e abusos que são teoricamente infinitos, mas que na realidade compreendem a uma gama de bases de poder que existe naquele determinado momento e que estruturam e distribuem ao longo de um espectro do tipo dominantes/ marginais os significados das histórias produzidas (JENKINS, 2005, p. 52).

Admitindo que os "acontecimentos" do passado, objeto central de estudo da História, podem ter vários significados dependendo de como são registrados, interpretados e transmitidos, trabalhar a partir das versões de pessoas que participaram, efetivamente, desses 
"acontecimentos", pode apresentar-se como uma interessante intervenção para (e sobre) a produção de conhecimento, aceitando-se, na base dessa opção, a possibilidade de que vivenciamos e registramos "alguma" História e não "a" História com um significado unívoco, global e generalizante.

\section{Metodologia}

No presente texto, buscamos apresentar um estudo que mostra os rastros dos primeiros cursos que habilitavam o professor a ministrarem aulas de Matemática na cidade de Uberlândia nas décadas de 1960 e 1970.

Para nos auxiliar na elaboração desses resultados além dos documentos disponíveis tomamos por fonte de dados às edições da Revista Documenta que foram publicados nos anos de 1962 até 1980.

Essa revista apresenta assuntos relativos ao Conselho Federal de Educação, como pareceres dos conselheiros sobre pedidos de criação de cursos e instituições, posicionamentos dos conselheiros sobre aspectos da educação brasileira, explicações sobre normas e legislações (MARTIN-SALANDIM, 2012). Para servir como ilustração para o leitor, na figura 1, temos um mosaico de algumas capas das publicações da Revista Documenta, desde a primeira até a última edição publicada atualmente.

\section{Figura 1 - Capas da Revista Documenta}

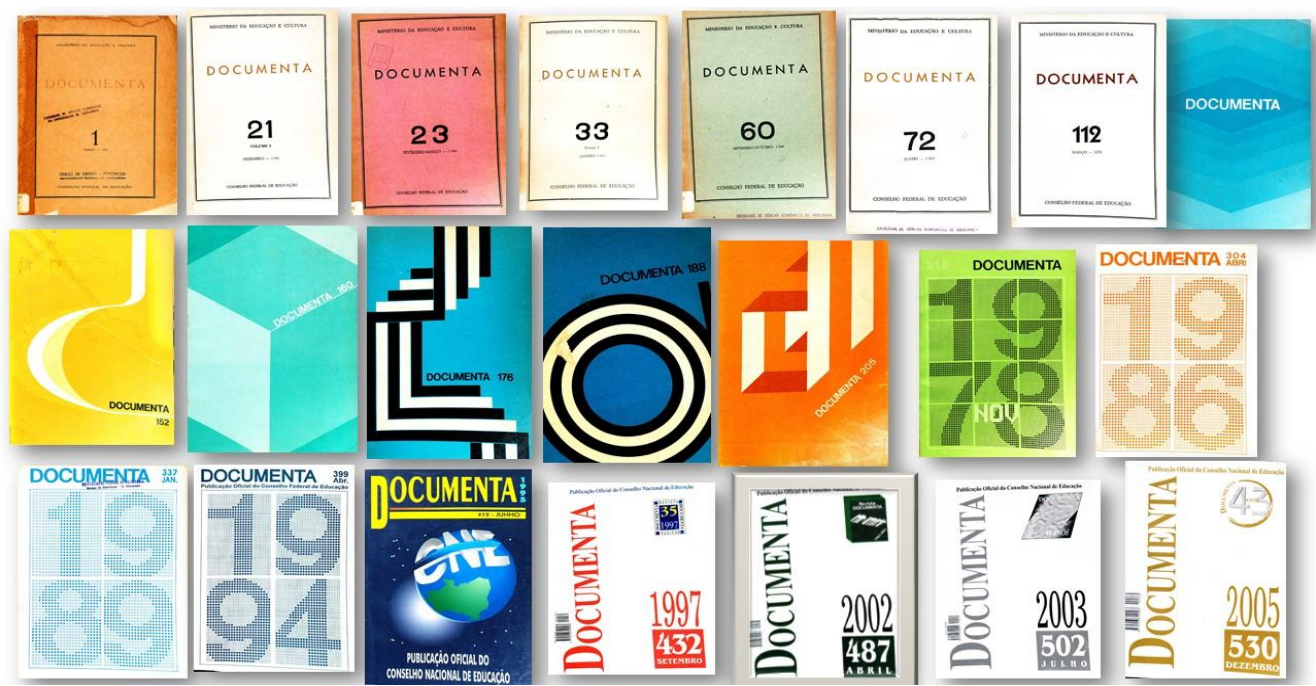

Fonte: Revista Documenta 
O levantamento de dados foi realizado junto ao acervo da Revista Documenta que está localizado na biblioteca da Universidade Federal de Uberlândia, Campus Santa Mônica. Todas as informações foram digitalizadas e arquivadas, depois foi elaborado um estudo sistemático tomando por base o uso de tabelas para o auxílio da compreensão e análise dos dados. Na sequência do texto, apresentamos uma discussão e síntese desses resultados.

\section{Rastros dos cursos em que habilita o professor a lecionar Matemática.}

Essa seção tem como propósito apresentar uma trajetória histórica dos cursos que habilitavam o professor a lecionar Matemática, nas décadas de 1960 a 1970, através da análise de alguns aspectos relacionados ao processo de criação desses cursos utilizando como fonte de dados, além das edições da revista Documenta que foram publicados nos anos de 1962 até 1979, a inserção de outros documentos disponíveis nos auxiliaram nesse processo.

No período em que este estudo pretende abordar encontramos na cidade de Uberlândia dois movimentos que deflagram os rastros do surgimento de cursos que habilitavam o professor a ministrarem aulas de Matemática.

O primeiro desses cursos nasce do encontro com a constituição das primeiras faculdades isoladas, quando 1960 é criada a Faculdade de Filosofia, Ciências e Letras de Uberlândia, com os cursos de Pedagogia e Letras, posteriormente, o curso de História e, em 1967 o curso de Matemática.

O outro curso para professores de Matemática sendo criado em 1975, como uma habilitação do curso de Ciências oferecido pela Faculdade de Educação, Ciências e Letras mantido pela Associação Brasil Central de Educação e Cultura.

Para auxiliar no entendimento da região em estudo, na figura 1, apresentamos um mapa localizando a cidade de Uberlândia frente ao estado de Minas Gerais e, consequentemente, no Brasil. 


\section{Figura 1 - Localização de Uberlândia}

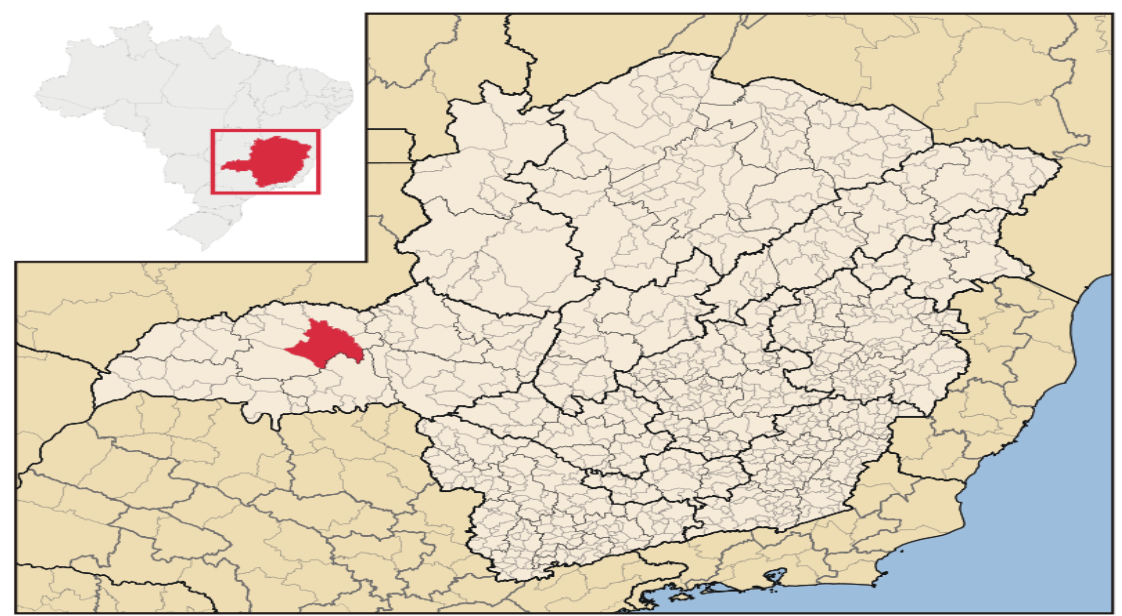

Fonte: www.skyscrapercity.com

O curso de Matemática da Faculdade de Filosofia, Ciências e Letras de Uberlândia foi criado em 1967, mas de acordo com Freitas (2000) esse curso recebeu autorização para funcionar a sua primeira turma apenas no início de 1968, isto ocorreu devido apenas dois alunos passarem no exame vestibular da época.

Esse curso inicialmente autorizado a funcionar com total de 2700 horas, correspondentes a 675 horas anuais "havendo condições adequadas, poderá a faculdade reduzir ou aumentar o tempo de duração dos cursos, obedecendo as normas da Portaria Ministerial $n^{\circ} 159 / 65$ e foi autorizado a funcionar com um total inicial de 50 vagas anuais" (DOCUMENTA 139, p. 72, 1972).

Além disso, foi autorizado ao curso funcionar com o seguinte currículo mínimo como podemos observar no quadro 1 .

Quadro 1: Currículo Mínimo

\begin{tabular}{|c|c|}
\hline Disciplinas Específicas & Disciplinas Pedagógicas \\
\hline Desenho Geométrico e Geometria Descritiva & Psicologia da Educação: Adolescência- \\
& Aprendizagem \\
\hline Fundamento de Matemática Elementar & Didática \\
\hline Física Geral & Elementos de Administração Escolar \\
\hline Cálculo Diferencial e Integral & Prática de Ensino sob a forma de Estágio \\
& Supervisionado \\
\hline
\end{tabular}




\begin{tabular}{|c|l|}
\hline Geometria Analítica & \\
\hline Álgebra & \\
\hline Cálculo Numérico & \\
\hline Estatística & \\
\hline
\end{tabular}

Fonte: Documenta n ${ }^{0} 139$, p. 73, 1972.

De acordo com a revista Documenta $\mathrm{n}^{\circ}$ 10, p. 99 estas disciplinas pedagógicas oferecidas pelo curso de Matemática pode ser verificada nas recomendações do Conselho Federal de Educação.

Nesse mesmo número da revista mais nas páginas 85 e 86 podemos identificar as disciplinas do currículo mínimo para o curso de Matemática, dessa forma é possível afirmar que o curso de Matemática segue a recomendação legal.

Na revista Documenta $n^{\circ} 139$, p. 73 é apontada que o curso de Matemática será de quatro anos e as disciplinas do currículo mínimo serão distribuídas como podemos ver a seguir no quadro 2. Uma observação, nesta época os anos de estudo eram chamados de séries, talvez por influência da escola básica, atualmente a distribuição é entendida por semestre.

Quadro 2: Distribuição das disciplinas ao longo do curso de Matemática

\begin{tabular}{|c|c|}
\hline Série & Disciplinas \\
\hline \multirow{4}{*}{$1^{\mathrm{a}}$} & 1. Desenho Geométrico e Geometria Descritiva \\
& 2. Fundamentos de Matemática Elementar \\
& 3. Cálculo Diferencial e Integral \\
4. Geometria Analítica \\
5. Introdução Geral à Filosofia
\end{tabular}




\begin{tabular}{|c|c|}
\hline $4^{a}$ & $\begin{array}{l}\text { 1. Física Geral } \\
\text { 2. Álgebra } \\
\text { 3. Cálculo Numérico } \\
\text { 4. Estatística } \\
\text { 5. Prática de Ensino sob a forma de estágio supervisionado } \\
\text { 6. Estrutura de Funcionamento do Ensino de } 2^{\circ} \mathrm{Grau}\end{array}$ \\
\hline
\end{tabular}

Fonte: Documenta ${ }^{0}$ 139, p. 73, 1972.

Podemos observar no quadro 2, que as disciplinas Fundamentos de Matemática Elementar, Cálculo Diferencial e Integral, Física Geral e Álgebra eram ministradas em três anos do curso de Matemática. E, em todos os semestres existiam as disciplinas de cunho pedagógico, no entanto, o que chama a atenção, é o fato de apenas no último ano do curso a presença da disciplina de Estágio Supervisionado, onde os estudantes têm o primeiro contato com a escola, a carga horária dessa disciplina era de apenas 120 horas.

No quadro 3, apresentamos a relação professor x disciplina e o parecer que autorizou a indicação destes professores. Também apresentamos para cada um dos professores $\mathrm{x}$ disciplinas o número da Documenta que aponta esta indicação.

Quadro 3: Relação de professores x disciplinas aprovados pelo CFE

\begin{tabular}{|c|c|c|c|}
\hline Professor & Disciplina & Parecer $n^{0}$ & Revista Documenta \\
\hline Ênio Vilela de Andrade & Geometria Analítica & $527 / 66$ & $\mathrm{n}^{\circ} 60$, p. 120 \\
\hline José Peppe Júnior & $\begin{array}{c}\text { Cálculo Diferencial e } \\
\text { Integral }\end{array}$ & $252 / 67$ & $\mathrm{n}^{\mathrm{o}} 72$, p. 31 \\
\hline Galba Gouveia Porto & $\begin{array}{l}\text { Desenho Geométrico e } \\
\text { Geometria Descritiva }\end{array}$ & $527 / 66$ & $\mathrm{n}^{\mathrm{o}} 60$, p. 11 \\
\hline $\begin{array}{l}\text { Celso Corrêa dos } \\
\text { Santos }\end{array}$ & Física Geral & $527 / 66$ & $\mathrm{n}^{\mathrm{o}} 60$, p. 12 \\
\hline Yone Vicentini Gomes & Álgebra & $527 / 66$ & $\mathrm{n}^{\circ} 60$, p. 12 \\
\hline $\begin{array}{c}\text { Marcia Augusta } \\
\text { Crosara Petronzio }\end{array}$ & $\begin{array}{c}\text { Fundamentos de } \\
\text { Matemática Elementar }\end{array}$ & $527 / 66$ & $\mathrm{n}^{\mathrm{o}} 60$, p. 12 \\
\hline $\begin{array}{l}\text { Luiz Arthur Meinberg } \\
\text { Santos }\end{array}$ & Cálculo Numérico & $227 / 67$ & $\mathrm{n}^{\mathrm{o}} 72$, p. 31 \\
\hline Mariú Cerchi Borges & Psicologia da Educação & $303 / 67$ & $\mathrm{n}^{\mathrm{o}} 74$, p. 115 \\
\hline Ilar Garotti & Didática Geral & $329 / 63$ e $363 / 67$ & $\mathrm{n}^{\circ} 21$, p. 34 e 76, p. 85 \\
\hline
\end{tabular}




\begin{tabular}{|c|c|c|c|}
\hline $\begin{array}{c}\text { Renato Campelo } \\
\text { Ribeiro }\end{array}$ & Estatística & $150 / 66$ e $98 / 69$ & $\mathrm{n}^{\circ} 44,47$ e 99 \\
\hline
\end{tabular}

Fonte: Documenta ${ }^{0} 139$, p. 74, 1972.

Destacamos que nesse documento apontado pela revista Documenta $\mathrm{n}^{\circ} 139$, de 1972, não é informado quem eram os professores que ministrariam as disciplinas de Prática de Ensino sob a forma de estágio supervisionado, Estrutura de Funcionamento do Ensino de $2^{\circ}$ Grau, Introdução a Filosofia e Estudos de Problemas Brasileiros.

Os dados nos apontam que o curso de Matemática, em seu início, tinha como professores aqueles formados em engenharia, isto se deve talvez ao fato de, à época, a faculdade de engenharia já estar estabelecida na cidade de Uberlândia.

Em 8 de novembro de 1972 pelo Decreto $\mathrm{n}^{\circ} 71.335^{6}$ foi concedido reconhecimento ao curso de Matemática da Faculdade de Filosofia, Ciências e Letras da Fundação Universidade de Uberlândia.

Em relação ao curso de Ciências com habilitação em Matemática, na Revista Documenta, $\mathrm{n}^{\circ} 147$, de 1973 , é apontado o requerimento a partir do processo $\mathrm{n}^{\circ}$ 203.731/72 a autorização para o funcionamento de uma Faculdade de Educação que seria mantido pela Associação Brasil Central de Educação e Cultura.

A proposta seria que esta Faculdade de Educação oferecesse, inicialmente para o seu funcionamento, os cursos de Pedagogia, com habilitações em Orientação Educacional, Administração e Inspeção e Supervisão Escolar de $1^{\circ}$ e $2^{\circ}$ graus, Licenciaturas Polivalentes em Letras, Ciências e Estudos Sociais e Licenciaturas de $1^{\circ}$ e $2^{\circ}$ graus em Física, Química e Matemática.

Após cumprir todas as exigências pedidas pelo Conselho Federal de Educação (CNE), foi pelo Decreto $n^{\circ} 76.064^{7}$, de 31 de julho de 1975, que este mesmo Conselho, autorizou o funcionamento da Faculdade de Educação, Ciências e Letras, com os cursos de Pedagogia, de Letras, de Ciências (Polivalentes) e de Estudos Sociais.

Em relação ao curso de Matemática, até então era oferecido como uma habilitação do curso de Ciências, nas chamadas Ciências Polivalentes, onde se oferecia a Licenciatura de $1^{\circ}$ Grau e a Licenciatura em Matemática.

\footnotetext{
${ }^{6}$ Documenta ${ }^{\circ} 144$, p. 404,1972

${ }^{7}$ Documenta ${ }^{\circ} 176$, p. 381,1975
} 
Nessas habilitações, os estudantes que ingressavam no curso de Ciências cursavam inicialmente as disciplinas do ciclo básico que eram comuns a todos e, depois em outra fase do curso cursaria as disciplinas específicas da área de Matemática.

O currículo do curso de Licenciatura de $1^{\circ}$ Grau era desenvolvido em um mínimo de seis semestres letivos (ou três anos), com um total de 1.944 horas/aula, sendo 108 destinadas à Educação Física, distribuídas nos termos das normas vigentes, e 188 horas de Prática de Ensino de Ciências ao nível de $1^{\circ}$ grau.

Para se obter o diploma de Licenciado em Matemática, o estudante daria continuidade à Licenciatura de $1^{\circ}$ grau, por mais quatro semestres letivos no mínimo, perfazendo um acréscimo de 1.440 horas/aula. Totalizando assim 3.384 horas/aula, sendo 108 de disciplinas obrigatórias, tendo a duração mínima de 10 semestres letivos ou o equivalente a 5 anos.

Em relação ao corpo docente, o curso de Ciências possuía uma variedade muito grande de profissionais das mais diferentes áreas do conhecimento associados às habilitações que eram oferecidas.

No documento inicial que foi encaminhado para o CNE, do total de professores pertencentes ao quadro docente do curso de Ciências seis deles eram relacionados diretamente às disciplinas especificas para ministrar aulas na habilitação em Matemática, a saber: Ana Beatriz Lóes Cicci de Castro responsável por ministrar as disciplinas de Matemática, Cálculo Diferencial e Integral e Geometria Analítica, o professor Sebastião Ribeiro Ferreira era o responsável pelas disciplinas Cálculo Diferencial e Integral, Físico-Química e Química. Já o professor Julmar de Oliveira Diniz ministraria Cálculo Diferencial e Integral, Geometria Analítica e Probabilidade e Estatística, o professor Carlos José Borges seria o responsável pelas disciplinas de Análise Matemática e Matemática Aplicada. A professora Maria Cristina Pereira Braga ficou responsável pela disciplina de Prática de Ensino e, por fim, a professora Onélia Marçal Mattar pelas disciplinas de Álgebra, Análise Matemática e Processamento de Dados.

Os professores citados anteriormente tinham a formação inicial em engenharia ou em Licenciatura em Matemática. Os professores licenciados também eram docentes nas escolas de educação básica.

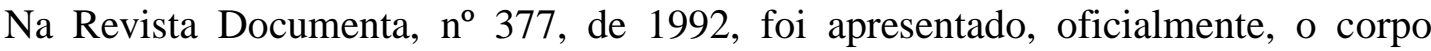
docente que iria ministrar as disciplinas do curso de Licenciatura em Matemática, a formação 
desses profissionais eram as seguintes: com mestrado - cinco professores, mestrandos - três professores, com especialização - quatro professores e por fim, com graduação - cinco professores.

Em 3 de fevereiro de 1993 pela Portaria ${ }^{\circ} 175$ publicado na Revisa Documenta $n^{\circ}$ 386, de 1993, foi reconhecido o curso de habilitação em Matemática, licenciatura plena, ministrada pela Faculdade de Educação, Ciências e Letras de Uberlândia.

\section{CONSIDERAÇÕES FINAIS}

Apresentamos nesse artigo um estudo cujo cenário de investigação foram os primeiros cursos de formação dos professores de Matemática na cidade de Uberlândia, nas décadas de 1960 e 1970.

Esses resultados, mesmo que incipientes, nos instigam ao delineamento mais detalhado da formação dos professores nessa região, e nos apontam que os próximos passos dessa pesquisa na busca de depoentes que vivenciaram esse período de desenvolvimento da difusão cultural, educacional e econômica não apenas na cidade de Uberlândia mais também, no Triângulo Mineiro, se torna viável e imprescindível.

Assim, destacamos que a expectativa desse estudo está em contribuir com os pesquisadores que tem interesse na Historia da Educação Matemática, na história da constituição da formação do professor (de Matemática) nessa região de inquérito, no currículo dos cursos da época e, com um projeto maior que é o do Mapeamento da Formação e Atuação de Professores de Matemática no Brasil, desenvolvido pelo Grupo História Oral e Educação Matemática - Ghoem.

\section{REFERÊNCIAS}

ALBUQUERQUE JÚNIOR, D. M. História: a arte de inventar o passado - Ensaios de teoria da história. 1. ed. Bauru: EDUSC, 2007.

ABBAGNANO, N. Dicionário de Filosofia. 5º ed. São Paulo: Martins Fontes, 2007.

ALMEIDA, S. P. N. C. Um lugar: muitas histórias - o processo de formação de professores de Matemática na região de Montes Claros, norte de Minas Gerais (19601990). Tese (Doutorado) - UFMG, Belo Horizonte, 2015. 
BARALDI, I. M. Retraços da Educação Matemática na região de Bauru (SP): uma história em construção. Tese (Doutorado) - UNESP, Rio Claro, 2003.

BRASIL. Ministério da Educação. Governo Federal. Decreto no 50.163, de 28 de janeiro de 1961. 1961. Disponível em <http://www2.camara.leg.br/legin/fed/decret/1960-1969/decreto50163-28-janeiro-1961-389980-publicacaooriginal-1-pe.html> Acesso em: 26 set. 2013.

BRASIL. Ministério da Educação. Governo Federal. Decreto $\mathbf{n}^{\mathbf{0}}$ 60.771, de 30 de maio de 1967. 1967. Disponível em <http://www2.camara.leg.br/legin/fed/decret/1960-1969/decreto60771-30-maio-1967-402000-publicacaooriginal-1-pe.html > Acesso em: 26 set. 2013.

BOTH, B. C. Sobre a formação de professores de matemática em Cuiabá- MT (19601980). Dissertação (Mestrado) - UNESP, Rio Claro, 2014.

CAETANO, C.G; DIB, M.M.C. A UFU no imaginário social. Uberlândia: Universidade Federal de Uberlândia, 1988.

CURY, F. G. Uma história da formação de professores de Matemática e das Instituições formadoras do estado do Tocantins. Tese (Doutorado) - UNESP, Rio Claro, 2011.

CURY, F. G. Uma narrativa sobre a formação de professores de Matemática em Goiás. Dissertação (Mestrado) - UNESP, Rio Claro, 2007.

DOCUMENTA. Rio de Janeiro: Conselho Federal de Educação. 1962 até 1999.

FERNANDES, D. N. Sobre a formação do professor de Matemática no Maranhão: cartas para uma cartografia possível. Tese (Doutorado) - UNESP, Rio Claro, 2011.

FERREIRA, A.C; BRITO, A.J; MIORIM, M.A. História de formação de professores que ensinaram matemática no Brasil. Campinas: Ílion, 2012.

FAORO, T.C.T. A formação de professores de matemática da Universidade Federal de Mato Grosso do Sul: um olhar sobre os anos iniciais da licenciatura em Dourados. Dissertação (Mestrado) - UFMS, Campo Grande, 2014.

FILLOS, L. M. A Educação Matemática em Irati (PR): memórias e história. Dissertação (Mestrado) - UFPr, Curitiba, 2008.

GARNICA, A. V. M. Cartografias contemporâneas: mapa e mapeamento como metáforas para a pesquisa sobre formação de professores de Matemática. ALEXANDRIA Revista de Educação em ciências e Tecnologia, v.6, n.1, p. 35- 60, 2013.

Cartografias contemporâneas: mapeando a Formação de Professores de Matemática no Brasil. Curitiba: Appris, 2014.

GARNICA, A. V. M.; FERNANDES, D. N.; SILVA, H. Entre a amnésia e a vontade de nada esquecer: notas sobre regime de historicidade e história oral. Bolema (Rio Claro), v. 25, $\mathrm{n}^{\circ} 41$, p. 213-250, 2011. 
GARNICA, A.V.M.; SOUZA, L.A. Elementos de história da Educação Matemática. São Paulo: Cultura Acadêmica, 2012.

GOMES, A. R; WARPECHOWKI, E. M; NETTO, R. S. Fragmentos, imagens, memórias: 25 anos de federalização da Universidade Federal de Uberlândia. Uberlândia: EDUFU, 2003.

MACENA. M. M. M. Sobre formação e prática de professores de Matemática: estudo a partir de relatos de professores, década de 1960, João Pessoa (PB). Tese (Doutorado) UNESP, Rio Claro, 2013.

MARIANO DA SILVA, C. R. Uma, nove ou dez narrativas sobre as licenciaturas em Ciências e Matemática em Mato Grosso do Sul. Tese (Doutorado) - UNESP, Rio Claro, 2015.

MARTINS-SALANDIM, M. E. A interiorização dos cursos de Matemática no estado de São Paulo: um exame da década de 1960. Tese (Doutorado) - UNESP, Rio Claro, 2012.

MARTINS-SALANDIM, M. E. Escolas técnicas agrícolas e Educação Matemática: história, práticas e marginalidade. Dissertação (Mestrado) - UNESP, Rio Claro, 2007.

MORAIS, M. B. Peças de uma história: formação de professores de Matemática na região de Mossoró (RN). Dissertação (Mestrado) - UNESP, Rio Claro, 2012.

PINTO, A. H. Educação Matemática e formação para o trabalho: práticas escolares na escola técnica de Vitória - 1960 a 1990. Tese (Doutorado) - UNICAMP, Campinas, 2006.

SILVA, M. S. Sobre a formação de professores das séries iniciais na região de São José do Rio Preto-SP na ocasião dos centros específicos de formação e aperfeiçoamento para o magistério (CEFAM). Dissertação (Mestrado) - UNESP, Rio Claro, 2015.

TOILLIER, J. S. A formação do professor (de Matemática) em terras paranaenses inundadas. Dissertação (Mestrado) - UNESP, Rio Claro, 2013.

RECEBIDO EM: 02/03/2016

APROVADO PARA PUBLICAÇÃO EM: 29/11/2016 